\title{
Multi-laboratory study of the analysis and kinetics of stanozolol and its metabolites in treated calves $\dagger$
}

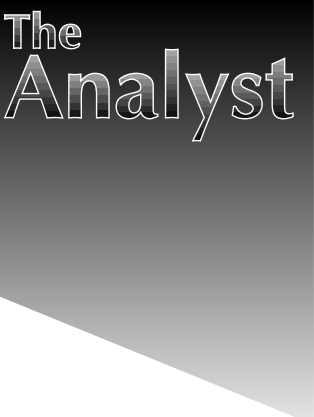

\author{
H. F. De Brabander, ${ }^{* a}$ K. De Wasch, ${ }^{a}$ L. A. van Ginkel, ${ }^{b}$ S. S. Sterk, ${ }^{b}$ M. H. Blokland, ${ }^{b}$ \\ Ph. Delahaut, ${ }^{c}$ X. Taillieu, ${ }^{c}$ M. Dubois, ${ }^{c}$ C. J. M. Arts, ${ }^{d}$ M. J. van Baak, ${ }^{d}$ L. G. \\ Gramberg, ${ }^{d}$ R. Schilt, ${ }^{e}$ E. O. van Bennekom, ${ }^{e}$ D. Courtheyn,$f$ J. Vercammen $f$ and R. F. \\ Witkamp $^{d g}$
}

\author{
a RUG, Faculty of Veterinary Medicine, Department of Veterinary Food Inspection, \\ Laboratory of Chemical Analysis, Salisburylaan, 133, B-9820 Merelbeke, Belgium. E-mail: \\ Hubert.DeBrabander@rug.ac.be \\ ${ }^{b}$ RIVM: European Union Community Reference Laboratory, A. Van Leeuwenhoeklaan 9, \\ P.O. Box 1, NL-3720 BA Bilthoven, The Netherlands \\ c CER, Laboratoire d'Hormonologie, Rue de Point du jour, 8 B-6900, Marloie, Belgium \\ ${ }^{d}$ TNO, Nutrition and Food Research Institute, Analytical Sciences Division, Utrechtseweg 48, \\ P.O. Box 360, NL-3700 AJ Zeist, The Netherlands \\ e RIKILT-DLO, Bornsesteeg, 45 P.O. Box 230, NL-6700 AE Wageningen, The Netherlands \\ $f$ ROL (State Laboratory), Braemkasteelstraat 59, B-9050 Gentbrugge, Belgium \\ g RU Utrecht, Veterinary Faculty Utrecht, The Netherlands
}

Received 2nd July 1998, Accepted 17th August 1998

\begin{abstract}
The European Union banned the use of anabolic steroids for cattle fattening in 1988. Analytical techniques able to detect trace amounts of the parent drugs and their metabolites are mandatory for the control of abuse. Stanozolol (Stan) is an anabolic steroid that is often found in injection sites and cocktails. However, it has never been detected in tissues (kidney fat, meat) or excreta (urine, faeces) taken during regulatory inspection. The difference between the structure of Stan and the other steroids (a pyrazole ring fused to the androstane ring system) is probably the cause of this phenomenon. In the multi-laboratory study described here, veal calves were treated with intramuscular doses of Stan. In the excreta of these calves the presence, absence and/or concentration of Stan and of its major metabolites 16ß-hydroxystanozolol and 3'-hydroxystanozolol were determined. For the determination of these analytes the different laboratories used different extraction and clean-up procedures and also evaluated different analytical techniques such as GC-MS (negative chemical ionization) and LC-MS-MS. The aim of this investigation was to explore which analyte should be validated for veterinary inspection purposes.
\end{abstract}

\section{Introduction}

In 1988 the European Union (EU) banned the use of anabolic steroids in animals intended for consumption. ${ }^{1}$ The Veterinary Food Inspectorate of each member state has to take a number of representative samples which are analysed in accredited field laboratories, controlled by a National Reference Laboratory (NRL). The NRLs are controlled by a Community Reference Laboratory (CRL). The list of steroids that might be misused in animal fattening is long, and new substances are regularly added to the list of target steroids. Some examples of newly introduced drugs are: norethandrolone, norgestrel and fluoxymesterone. These steroids are not necessarily strictly new. In order to mislead the inspection services, attempts are made to replace the so-called 'classical drugs' by unexpected or what are believed to be 'untraceable' drugs.

In this context, stanozolol is relatively 'old' (US patent granted in 1962; The Merck Index, 1996) ${ }^{2}$ and hitherto its abuse has mostly been untraceable except in injection sites taken at the slaughterhouse where it is often found. However, it has never been detected in tissues (kidney fat, meat) or excreta (urine, faeces) taken during regulatory inspection. Stanozolol $(5 \alpha-$ androstane-17 $\alpha$-methyl-17 $\beta$-ol[3,2-c]pyrazole)(Stan) most closely resembles methyltestosterone. Instead of the 3-keto

$\uparrow$ Presented at the Third International Symposium on Hormone and Veterinary Drug Residue Analysis, Bruges, Belgium, June 2-5, 1998. group, there is a pyrazole ring fused to the androstane ring system.

Stan cannot be detected with the test methods used for multiresidue analyses of steroids. In high-performance thin layer chromatography (HPTLC), ${ }^{3}$ the detection limit of Stan is inferior to the detection limit of most other steroids. Also, in gas chromatography-mass spectrometry (GC-MS) the drug has a different behaviour in comparison with that of related compounds. Moreover, the molecule is metabolised rapidly. In a related research area, sports doping, in both man and race horses, ${ }^{4}$ Stan has been the subject of metabolic research. The research group of the Deutsche Sporthochschule Koln, Germany, has studied and synthesised the urinary metabolites of Stan in man (Fig. 1). ${ }^{5-7}$

Other workers have used these metabolites in an effort to improve the GC-MS ${ }^{8,9}$ or LC-MS-MS ${ }^{10}$ methodology for the determination of these compounds in doping control. For veterinary inspection, it was demonstrated by GC-MS and LCMS that 16-hydroxystanozolol (16-OHStan) ${ }^{11,12}$ and 4,16-dihydroxystanozolol $(4,16 \text {-diOHStan })^{12}$ were the major metabolites of Stan in cattle. After some informal contacts between researchers in Belgium and The Netherlands, already involved in this subject, a working group on Stan was formed. At a meeting, during which methodology, results and ideas were exchanged, it was decided to perform an animal experiment on three veal calves, distribute the samples to the participating laboratories and have the results ready for the Third Inter- 
national Symposium on Hormone and Veterinary Drug Residue Analysis (the Ghent conference). The aim of this investigation was to explore which analyte should be validated for veterinary inspection purposes.

\section{Experimental}

\section{Apparatus}

The following apparatus was used: homogenisers (e.g. ultraturrax T25), centrifuges (e.g. Sorvall RC-26), rotary vacuum evaporators (e.g. Büchi R-114), water-baths (e.g. GFL), extraction flasks (250 and $500 \mathrm{ml})$, vacuum manifolds $(e . g$. Vacubrand ME2), nitrogen and other types of evaporator (e.g. Speedvac SC210A), chromatographic columns and tanks, autosampler vials (e.g. Chromacol N8-07), solid-phase extraction (SPE) $\mathrm{C}_{18}$ columns (e.g. $3 \mathrm{ml}$ Baker 7020-03), and standard laboratory equipment.

The different GC-MS and LC-MS apparatus used by the different laboratories is described below.

RUG (Faculty of Veterinary Medicine, Department of Veterinary Food Inspection, Laboratory of Chemical Analysis, Merelbeke, Belgium) and ROL (State Laboratory, Gentbrugge, Belgium). A Model P4000 pump (TSP, San Jose, CA, USA) coupled to an LCQ Ion Trap Mass Analyser (Finnigan-MAT, San Jose, CA, USA) with an electrospray interface and a Navigator 1.0 , respectively. Symmetry $C_{18}$ column $(5 \mu \mathrm{m}, 150 \times 2.1 \mathrm{~mm}$ id, Waters, Milford, MA, USA).

RIVM (European Union Community Reference Laboratory, Bilthoven, The Netherlands). An LC instrument equipped with a mass-selective detector (Hewlett-Packard, Palo Alto, CA, USA). Alltima $\mathrm{C}_{18}$ column $(5 \mu \mathrm{m}, 150 \times 2.1 \mathrm{~mm}$ id, Alltech, Arlington Heights, IL, USA). A gradient system was used. The mobile phase consisted of $0.5 \%$ ammonium acetatemethanol $(40+60$, to $20+80$ over $20 \mathrm{~min})$.

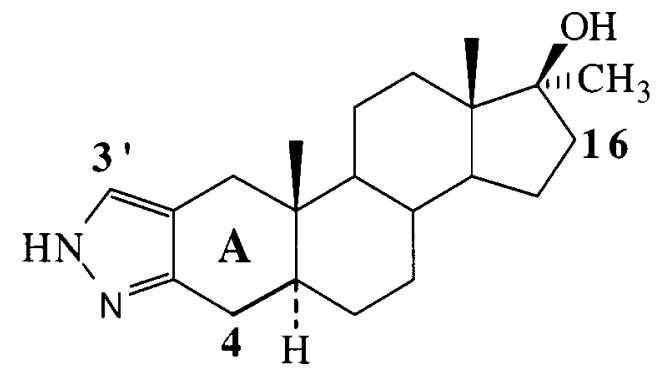

Stanozolol

(Stan)<smiles>Cc1n[nH]cc1C[C@@]1(C)C[C@H]2C1CC[C@@]1(C)C3C[C@@H](O)[C@@](C)(O)C3CCC21</smiles>

$16 \beta$-Hydroxystanozolol (16-OHStan)
CER (Laboratoire d'Hormonologie, Marloie, Belgium). A Quattro II mass spectrometer (Micromass UK, Altrincham, Cheshire, UK) consisting of a Hewlett-Packard 1100 liquid chromatograph and MS-MS Engine.

RIKILT-DLO: (Bornsesteeg, Wageningen, The Netherlands). A Hewlett-Packard Model 5989B MS Engine and a Model 5890 Series II Plus gas chromatograph.

\section{Reagents and reference compounds}

Most reference steroids were obtained from Steraloids (Wilton, NY, USA) or Sigma (St. Louis, MO, USA). The internal standard was stanozolol- $d_{3}$ ( $5 \alpha$-androstane- $17 \alpha$-methyl- $d_{3-}$ $17 \beta$-ol[3,2-c] pyrazole) (Stan-d ${ }_{3}$ ) (Sigma 9271) (for LC-MSMS). Stan was obtained from Sigma (Sigma 7132). The metabolites of Stan were obtained from Radian International (Austin, TX, USA): 3'-hydroxystanozolol (3-OHStan), $4 \beta$ hydroxystanozolol (4-OHStan), and 16 $\beta$-hydroxystanozolol (16-OHStan).

Helix pomatia juice (glucuronidase-sulfatase enzyme suspension) was obtained from Boehringer (Mannheim, Germany). All solvents used were of analytical-reagent grade from Merck (Darmstadt, Germany). Stock solutions of anabolic steroids were prepared at $200 \mathrm{ng} \mu \mathrm{l}^{-1}$ in ethanol. The stock solutions were diluted 10 -fold to give working solutions at a concentration of $20 \mathrm{ng} \mu \mathrm{l}^{-1}$.

\section{Clean-up procedures}

RUG. Urine $(10 \mathrm{ml})$ was centrifuged at $2700 \mathrm{~g}$ for $7 \mathrm{~min}$, and $3 \mathrm{ml}$ of $0.2 \mathrm{M}$ acetate buffer $(\mathrm{pH} 4,6)$ and $50 \mu \mathrm{l}$ of Helix pomatia juice were added. The mixture was incubated for $3 \mathrm{~h}$ at $55^{\circ} \mathrm{C}$ and then centrifuged at $2700 \mathrm{~g}$ for $10 \mathrm{~min}$. The solution was loaded on to an Extrelut column and extracted with diethyl ether $(3 \times 20 \mathrm{ml})$. The diethyl ether extracts were combined and evaporated to dryness. The residue was taken up in $50 \mu \mathrm{l}$ of ethanol and $10 \mu \mathrm{l}$ of the resulting solution were injected into the LC-MS system.<smiles>C[C@H]1c2n[nH]c(O)c2C[C@@]2(C)C1CC[C@@]1(C)C2CC[C@@H]1O</smiles>

3'-Hydroxystanozolol

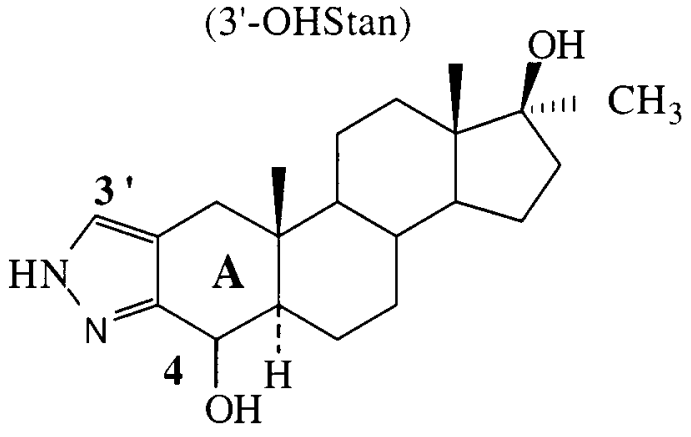

$4 \beta$-Hydroxystanozolol

(4-OHStan)

Fig. 1 Structures of stanozolol and its main urinary metabolites in man.5,6 
RIVM. Urine $(5 \mathrm{ml})$ was adjusted to $\mathrm{pH} 5.2$ and $40 \mu \mathrm{l}$ of Helix pomatia juice were added. The mixture was incubated overnight at $37{ }^{\circ} \mathrm{C}$ after which the solution was adjusted to $\mathrm{pH}$ 9 and extracted with hexane-butanol $(80+20, v / v)$. After evaporation of the extract to dryness, the residue was taken up in methanol-water $(80+20, \mathrm{v} / \mathrm{v})$. This solution was percolated through an SPE amino column and the column effluent was evaporated to dryness. The residue was taken up in methanolwater $(40+60, v / v)$ and $10 \mu l$ of the resulting solution were injected into the LC-MS system.

CER. To $10 \mathrm{ml}$ of urine were added $3 \mathrm{ml}$ of $2 \mathrm{~m}$ acetate buffer ( $\mathrm{pH}$ 4.6) and $100 \mu \mathrm{l}$ of Helix pomatia juice. The mixture was incubated for $3 \mathrm{~h}$ at $55^{\circ} \mathrm{C}$ and then centrifuged at $2700 \mathrm{~g}$ for 10 min. The solution was loaded on to an Extrelut column and extracted with diethyl ether $(3 \times 20 \mathrm{ml})$. The diethyl ether extracts were combined and evaporated to dryness. The residue was taken up in $50 \mu \mathrm{l}$ of ethanol and the resulting solution was subjected to an additional HPLC clean-up step.

RIKILT-DLO. Urine ( $10 \mathrm{ml})$ was adjusted to $\mathrm{pH} 4.8$ and 15 $\mu 1$ of Helix pomatia juice (Merck 4114) were added. The mixture was incubated for $16 \mathrm{~h}$ at $37^{\circ} \mathrm{C}$ after which the solution was cleaned-up on an ASPEC system with $\mathrm{C}_{18}$ and $\mathrm{NH}_{2}$ columns before evaporation to dryness. The residue was taken up in methanol-water $(38+62$, v/v $)$ and the resulting solution was cleaned-up by an HPLC procedure. The final extract was derivatised to 1-HFB-2-TMS ether (hexafluorobutyrate-trimethylsilyl) derivatives. An aliquot $(2 \mu \mathrm{l})$ was injected into the GC system.

ROL. Urine $(5 \mathrm{ml})$ was hydrolysed overnight at $52{ }^{\circ} \mathrm{C}(50 \mu \mathrm{l}$ of Helix pomatia; $\mathrm{pH}$ 5.2). The solution was cleaned-up on a $\mathrm{C}_{18}$ column [conditioning: $5 \mathrm{ml}$ of methanol and $5 \mathrm{ml}$ of water; washing: $10 \mathrm{ml}$ of water; elution: methanol $(3 \times 1 \mathrm{ml})]$. After evaporation of the eluate, the residue was dissolved in $100 \mu \mathrm{l}$ of water followed by $2 \mathrm{ml}$ of $0.1 \mathrm{M}$ phosphate buffer ( $\mathrm{pH}$ 6.0). The solution was applied to a Clean Screen Dau column [conditioning: $2 \mathrm{ml}$ of methanol, $2 \mathrm{ml}$ of water and $2 \mathrm{ml}$ of $0.1 \mathrm{M}$ phosphate buffer ( $\mathrm{pH}$ 6.0); washing: $1 \mathrm{ml}$ of $1 \mathrm{M}$ acetic acid, immediately followed by $6 \mathrm{ml}$ of methanol; elution: $6 \mathrm{ml}$ of ethyl acetate$28 \%$ ammonia solution $(97+3, \mathrm{v} / \mathrm{v})]$.

\section{GC-MS and LC-MS conditions}

RUG and ROL. The instrument was operated in the MS-MS positive ion mode. The maximum injection time was set at 400 ms with a total of $2 \mu$ s per scan. An electrospray voltage of 4.5 $\mathrm{kV}$ and a capillary temperature of $220^{\circ} \mathrm{C}$ were used. A methanol-1\% aqueous acetic acid solution $(70+30$, v/v) was used as the mobile phase at a flow rate of $0.3 \mathrm{ml} \mathrm{min}^{-1}$.

The time axis was divided into two segments. In the first segment, the three metabolites eluted; in the second segment, Stan and the internal standard co-eluted. The second segment was sub-divided into two scan events.

Segment 1: parent ion: $\mathrm{m} / \mathrm{z} 345$; isolation width: 1 ; collision energy: $28 \%$; mass range: $100-350 \mathrm{u}$.

Segment 2: scan event 1: parent ion: $\mathrm{m} / \mathrm{z}$ 329.2; isolation width: 1; collision energy: 28\%; mass range: 100-350 u; scan event 2: parent ion: $\mathrm{m} / \mathrm{z}, 332.2$; isolation width: 1 ; collision energy: 29\%; mass range: 100-350 u (see Table 1).

RIVM. The instrument was operated in the positive ion mode with atomospheric pressure chemical ionization (APCI) with a capillary voltage of $3000 \mathrm{~V}$ and a corona current of $10 \mu \mathrm{A}$. The fragmenter was set at $150 \mathrm{~V}$. The mass fragments monitored were $m / z 345$ for 3'-OHStan and 16-OHStan, $m / z 345$ and 327 for 4-OHStan and $m / z, 329$ for Stan. The peaks were baselineseparated.
CER. The instrument was operated in the APCI-MS-MS positive ion mode.

RIKILT-DLO. GC-MS conditions: from $130{ }^{\circ} \mathrm{C}$ (held for 2 $\min$ ) to $300{ }^{\circ} \mathrm{C}$ at $18.5^{\circ} \mathrm{C} \mathrm{min}-1$; injector temperature: $250{ }^{\circ} \mathrm{C}$ (splitless injection $2 \mathrm{~min}$ ), constant He flow of $0.84 \mathrm{ml} \mathrm{min}^{-1}$. Column: CP-Sil 5CB low bleed MS $(30 \mathrm{~m} \times 0.25 \mathrm{~mm}$ id, film thickness $0.25 \mu \mathrm{m})$. Acquisition method: selected ion monitoring on the ions at $\mathrm{m} / \mathrm{z}, 664,665,666,667,646,575,591$ and 179.

\section{Animal experiments}

These were carried out by TNO (Nutrition and Food Research Institute, Analytical Sciences Division, Zeist, The Netherlands) in co-operation with RU Utrecht (Veterinary Faculty Utrecht, The Netherlands). Three male calves of $c a$. 10 months (weight ca. $250 \mathrm{~kg}$ ) were housed in ventilated stables. Blank samples of urine, faeces and blood were taken. Subsequently, the animals were injected with a single intramuscular dose of $200 \mathrm{mg}$ of Stan suspended in $3 \mathrm{ml}$ of arachisoil. Blood samples were collected daily for $5 \mathrm{~d}$ while faeces and urine samples were collected daily for $14 \mathrm{~d}$ and frozen at $-24{ }^{\circ} \mathrm{C}$ until analysis. The samples were divided between the participating laboratories.

\section{Results and discussion}

\section{Current situation in the control of stanozolol abuse}

In injection sites Stan can be detected by both HPTLC and GCMS. ${ }^{13,14}$ A survey of Belgian injection sites demonstrated an increasing use of Stan from 1989 on to $1994 .{ }^{15,16}$ In 1995 in 141 positive injection sites Stan was found 72 times. ${ }^{17}$ However, Stan has never been detected in other matrices. In earlier investigations, ${ }^{11,12}$ it was found that Stan is metabolised rapidly so that the levels of the parent compound in the matrices hitherto examined (urine) are very low. It was also noted that the detection power of GC-MS for 16-OHStan is inferior to that for 3'-OHStan: for the injection of equal amounts of derivatised standards, a much ( $\mathrm{ca}$. ten times) lower signal was obtained for 16-OHStan than for $3^{\prime}$-OHStan. In LC-MS-MS, the detection power for 16-OHStan is not inferior to that for 3'-OHStan: the LC-MS-MS signals obtained after injection of equal amounts of standards are of the same magnitude. The necessity for derivatisation of the sample for GC-MS may explain this phenomenon. Steric hindrance of the hydroxy groups at the 16 and 17 positions may be the reason for the incomplete derivatisation.

\section{Detection of stanozolol and its metabolites}

Each participating laboratory developed a method using the same analytes, starting from a small amount of urine (5-10 ml), and adapted the clean-up to the instrument used. The individual

Table 1 Ions for LC-MS-MS of stanozolol and its metabolites

\begin{tabular}{|c|c|c|c|c|c|}
\hline Component & $\begin{array}{l}\text { Parent ion } \\
\left(\mathrm{MH}^{+}\right)\end{array}$ & Daughter ions & & & \\
\hline Stanozolol (Stan) & 329 & 311 & & 229 & 121 \\
\hline Stanozolol- $\mathrm{d}_{3}\left(\operatorname{Stan}-\mathrm{d}_{3}\right)$ & 332 & 314 & & 232 & 124 \\
\hline 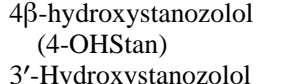 & 345 & 327 & 309 & & \\
\hline $\begin{array}{l}\text { (3'-OHStan) } \\
\text { 16ß-Hydroxystanozolol }\end{array}$ & 345 & 327 & & 229 & (159) \\
\hline (16-OHStan) & 345 & 327 & 309 & 227 & 159 \\
\hline
\end{tabular}


methods and results are presented below. An overview of the results in the form of a plot of the concentration found versus time is given later in Fig. 7.

RUG. In contrast to earlier results, ${ }^{11}$ a poor recovery of 16-OHStan was obtained using immunoaffinity chromatography (IAC). In co-operation with CER it was found that the IAC column had a lower affinity for 16-OHStan than for Stan and the other metabolites. Since 16-OHStan was known to be the major metabolite in cattle urine, another clean-up procedure was evaluated, viz., extraction with diethyl ether on an Extrelut column prior to LC-MS-MS analysis of 16-OHStan. Surprisingly, this approach was successful: in all veal urine samples, except for the blank 16-OHStan could be detected. No other metabolites were detected and only small amounts of Stan were observed in samples taken a few hours after injection. In Fig. 2 chromatograms and MS-MS scans of 16-OHStan and the internal standard for a urine sample taken $3 \mathrm{~d}$ after injection are shown (animal 1321). The MS-MS scan of 16-OHStan shows (too) many diagnostic ions $(n>20)$. This is an advantage for the fulfilment of quality criteria according to EC 93/256. ${ }^{18}$ However, extensive fragmentation is not favorable as regards the limit of detection. The limit of detection was not calculated but was estimated on the basis of spiked samples to be 0.5 $\mu \mathrm{g} \mathrm{kg}-1$.

The quantitative results are of the same magnitude as those found in the other laboratories (see Fig. 7). However, it was observed that the hydrolysis step with Helix pomatia juice has an adverse influence on the condition of the sample, making it less 'clean'.

RIVM. In this laboratory also a very simple clean-up procedure was developed: after hydrolysis, the metabolites were extracted with hexane-butanol $(80+20, \mathrm{v} / \mathrm{v})$. After percolation through an SPE amino column, the sample was ready for injection. By means of this method, 16-OHStan was also detected in all samples, except for the blank. In Fig. 3 chromatograms of 16-OHStan, Stan and the internal standard Stan- $\mathrm{d}_{3}$ are shown.

As can be seen in Fig. 3, no signal, except for that of the internal standard, was observed in the blank. In the urine sample taken shortly after injection (4B), both 16-OHStan and Stan itself were observed. In the urine sample taken $8 \mathrm{~d}$ after injection (animal 1321), no signal for Stan is present but a significant signal for 16-OHStan can be seen.

In contrast to the results of RUG and ROL, the use of a massselective detector in the APCI mode produces only one ion: the molecular ion. Hence, this method is only valid as a screening method since the criteria of EC93/256 are not fulfilled. The quantitative results are of the same magnitude but tend to be slightly lower than those found in the other laboratories (see Fig. 7).

CER. This laboratory used the same extraction on an Extrelut column as the RUG, but performed an additional clean-up by HPLC. With this method, 16-OHStan was detected and quantified by LC-MS-MS in all urine samples taken after
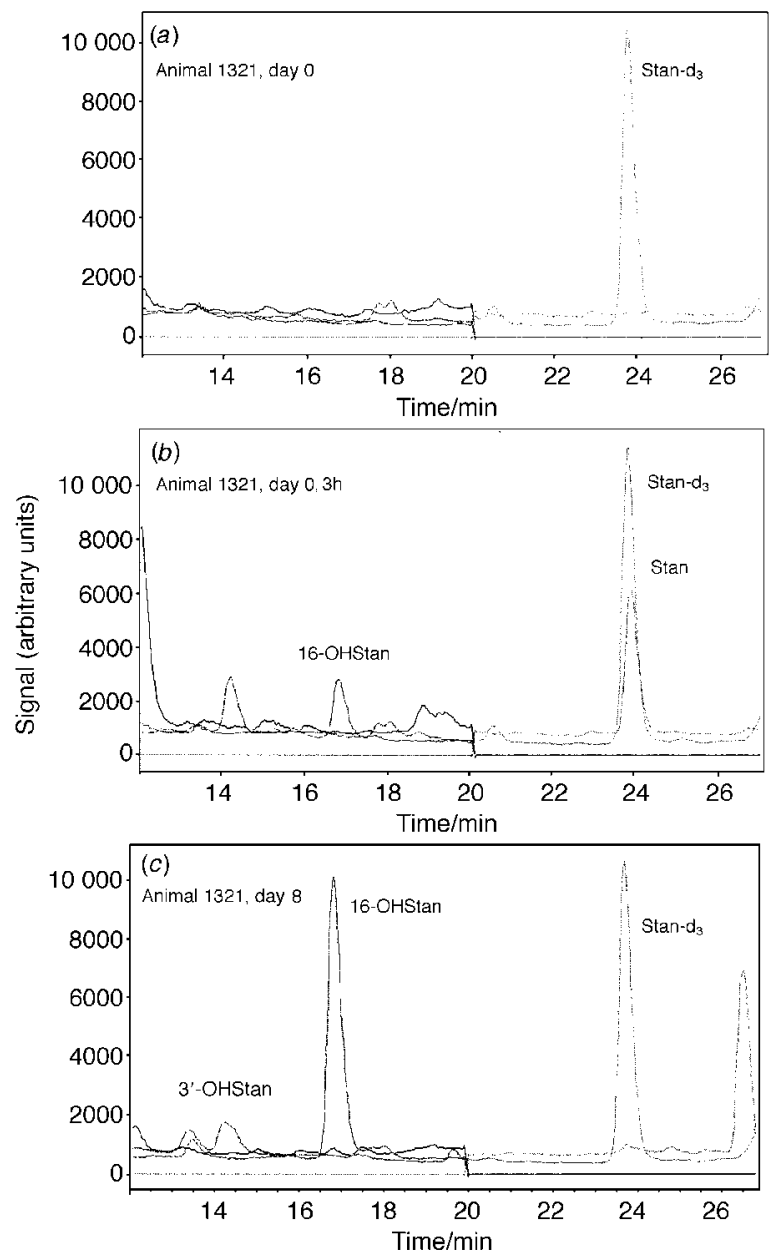

Fig. 3 Chromatograms of 16-OHStan, Stan and the internal standard Stan$\mathrm{d}_{3}$. (a) Blank; (b) $3 \mathrm{~h}$ after injection; (c) $8 \mathrm{~d}$ after injection (animal 1321) (data RIVM).
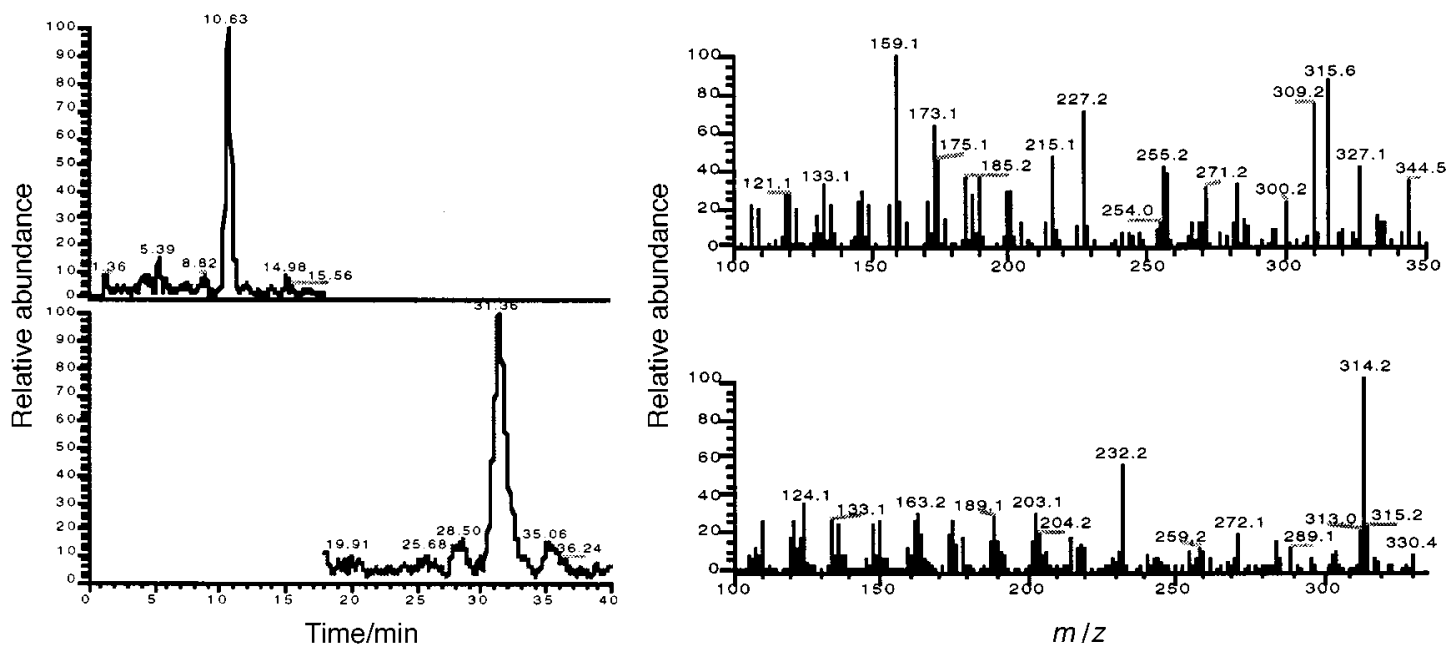

Fig. 2 Chromatograms and MS-MS scans of 16-OHStan and the internal standard Stan- $\mathrm{d}_{3}$ in a urine sample taken $5 \mathrm{~d}$ after injection (animal 1321) (data RUG). 
treatment of the animals. In Fig. 4 the MS and MS-MS scans (parent ion at $m / z, 345$ ) of 16-OHStan are shown. In order to improve the detection power, the chromatogram was recorded with one daughter ion $(81 \mathrm{u})$. In order to fulfil quality assurance (QA) criteria, ${ }^{18}$ in suspect samples more ions such as those at $\mathrm{m} / \mathrm{z}$ 93, 95109 and 121 could be used. In comparison with the LCQ instrument (Fig. 2 and 6), the MS-MS fragmentation obtained with the Quattro II instrument is totally different: there are fewer daughter ions and they are all in the lower mass region.

The CER also detected small amounts of $3^{\prime}$-OHStan in most of the urine samples. However, the concentrations found were lower than those of 16-OHStan.

RIKILT-DLO. This laboratory chose for GC-MS with negative chemical ionisation (NCI) for the detection of Stan and its metabolites after clean-up by SPE on an ASPEC system followed by HPLC fractionation (Stan, 16-OHStan and 3'OHStan are in different fractions). In Fig. 5 a chromatogram and a mass spectrum of 16-OHStan as its HFB-TMS derivative are given.

With this technique, 16-OHStan was detected in all the urine samples from the treated veal calves. In Fig. 5 also an ion chromatogram of 16-OHStan (ca. $8 \mu \mathrm{g} \mathrm{kg}^{-1}$ ) in the urine of animal 1899 is given. The metabolite $3^{\prime}$-OHStan could not be measured with this method because of an interfering peak. However, RIKILT noted that the GC-MS system was very sensitive to the loss of 16-OHStan in comparison with the response of 3'-OHStan, which stayed relatively constant. For the analysis of each series of samples, part of the column must be removed. These findings are analogous to earlier results ${ }^{11}$ from CER and RUG.

ROL. This laboratory evaluated and modified the clean-up procedure described by Ferchaus et al. ${ }^{12}$ in combination with LC-MS-MS.In Fig. 6 a four ion chromatogram (ions at $m / z, 159$, 201, 227 and 309) and an MS-MS scan (parent ion at $m / z$ 345) of 16-OHStan in the urine of animal 1321 taken $8 \mathrm{~d}$ after injection are given. The concentration found was $c a .10$ $\mu \mathrm{g} \mathrm{kg}-1$.

Shortly after injection of the animals ( $>10 \mathrm{~h}$ ), a substantial amount of Stan was also detected using the same method (Fig. $6)$.

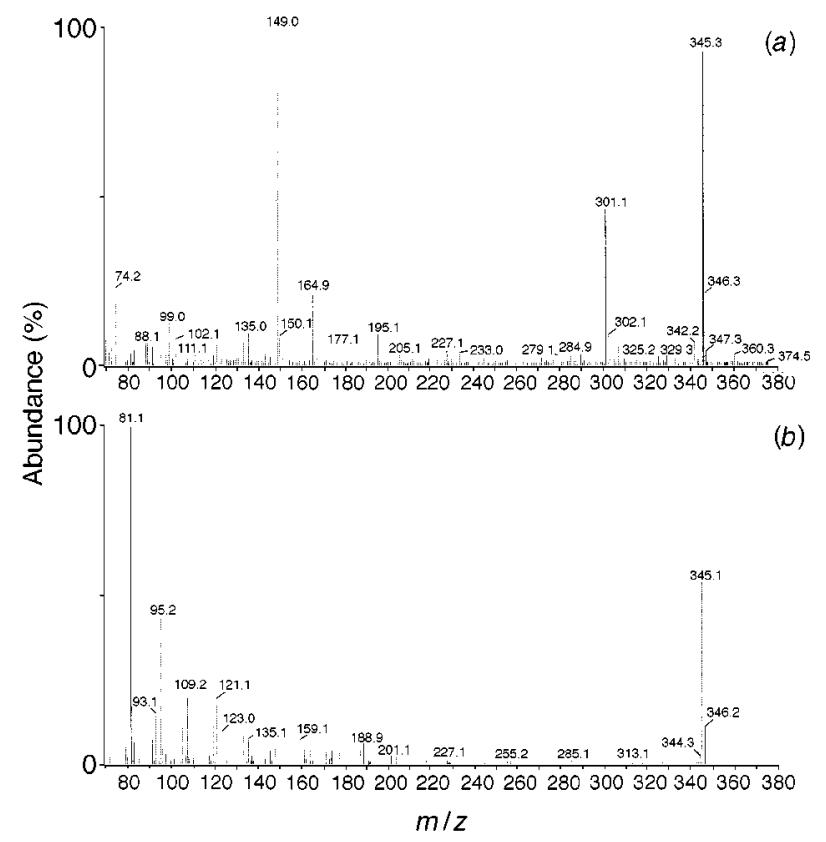

Fig. 4 MS scan (a) and MS-MS scan (parent ion at $\mathrm{m} / \mathrm{z}$ 345) (b) of 16-OHStan recorded on the Quattro II instrument (data CER).
The results of all the laboratories for one animal (1321) are given in Fig. 7. For the other two animals, analogous results were found. As can be seen in Fig. 7, the time course of the 16-OHStan concentration in urine was similar in all five laboratories.

In all laboratories 16-OHStan was detected at concentrations varying from 1 to $4 \mu \mathrm{g} \mathrm{kg}^{-1}$ during the first $4 \mathrm{~d}$ after injection. Subsequently, the 16-OHStan concentration increased to between 5 and $11 \mu \mathrm{g} \mathrm{kg}^{-1}$ (result depending on the laboratory). Later, the concentration decreased but was still detectable $14 \mathrm{~d}$ after the injection of Stan.

\section{Conclusion}

This interlaboratory study has shown that 16-OHStan is the major metabolite found in veal calf urine. This agrees with earlier findings of French ${ }^{12}$ and Belgian ${ }^{11}$ groups working on Stan. All methods used started from small amounts of urine $(5-10 \mathrm{ml})$ and used different clean-up systems. In addition to 16-OHStan, small amounts of Stan were detected in the first few hours after injection. The metabolite $3^{\prime}$-OHStan was only detected in trace amounts by some laboratories. The presence of the metabolite 4,16 -diOHStan ${ }^{12}$ was not studied because no standard was available at the time.

The metabolite 16-OHStan can be detected in urine directly after administration up to at least $14 \mathrm{~d}$ after injection of $200 \mathrm{mg}$ of Stan. A maximum is found in all animals between 8 and 10 $\mathrm{d}$ after injection. Although the methods were not fully compared with each other, LC-MS-MS seems to be the method of choice for the determination of this component. The limit of detection of the LC-MS methods was estimated to be $0.5-1 \mu \mathrm{g} \mathrm{kg}^{-1}$. Only one laboratory used GC-MS in the NCI mode. Although it was
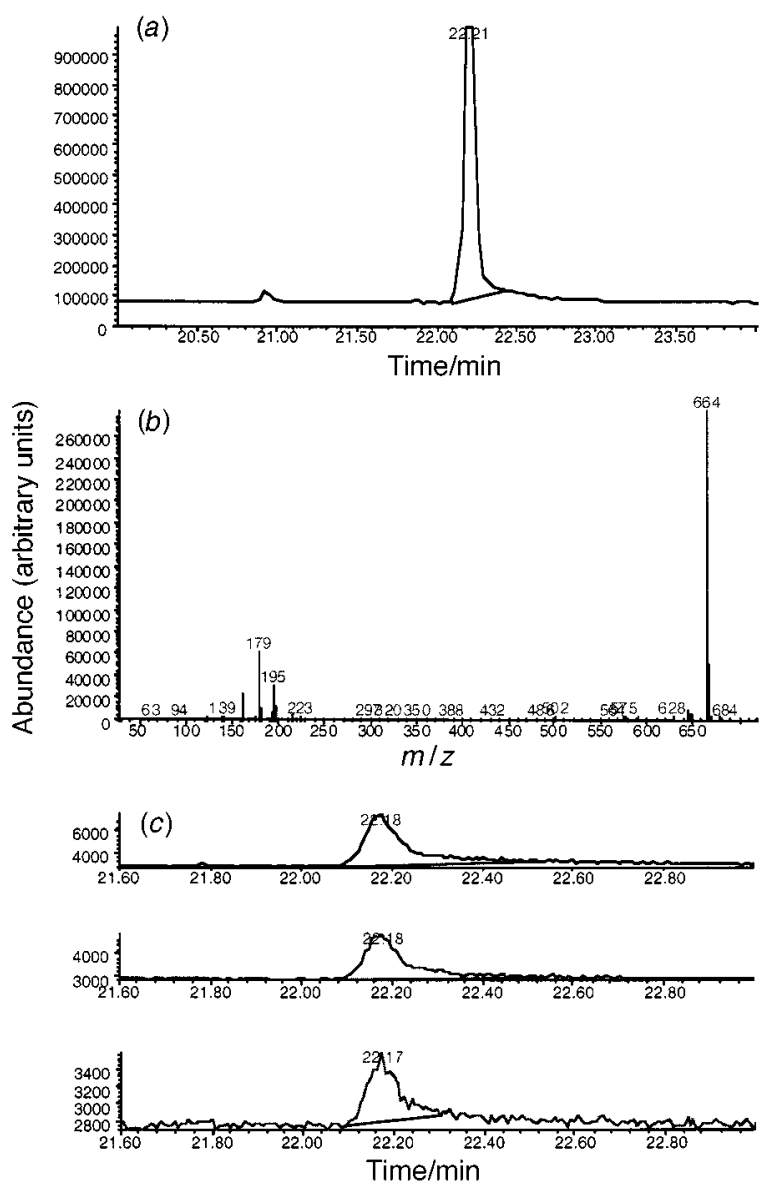

Fig. 5 Chromatogram $(a)$ and mass spectrum $(b)$ of a 16-OHStan standard (10 ng $\mu 1^{-1}$ ) (HFB-TMS derivative). Ion chromatogram (c) of 16-OHStan (ca. $8 \mathrm{ppb}$ ) in the urine of animal 1899 (data RIKILT). 

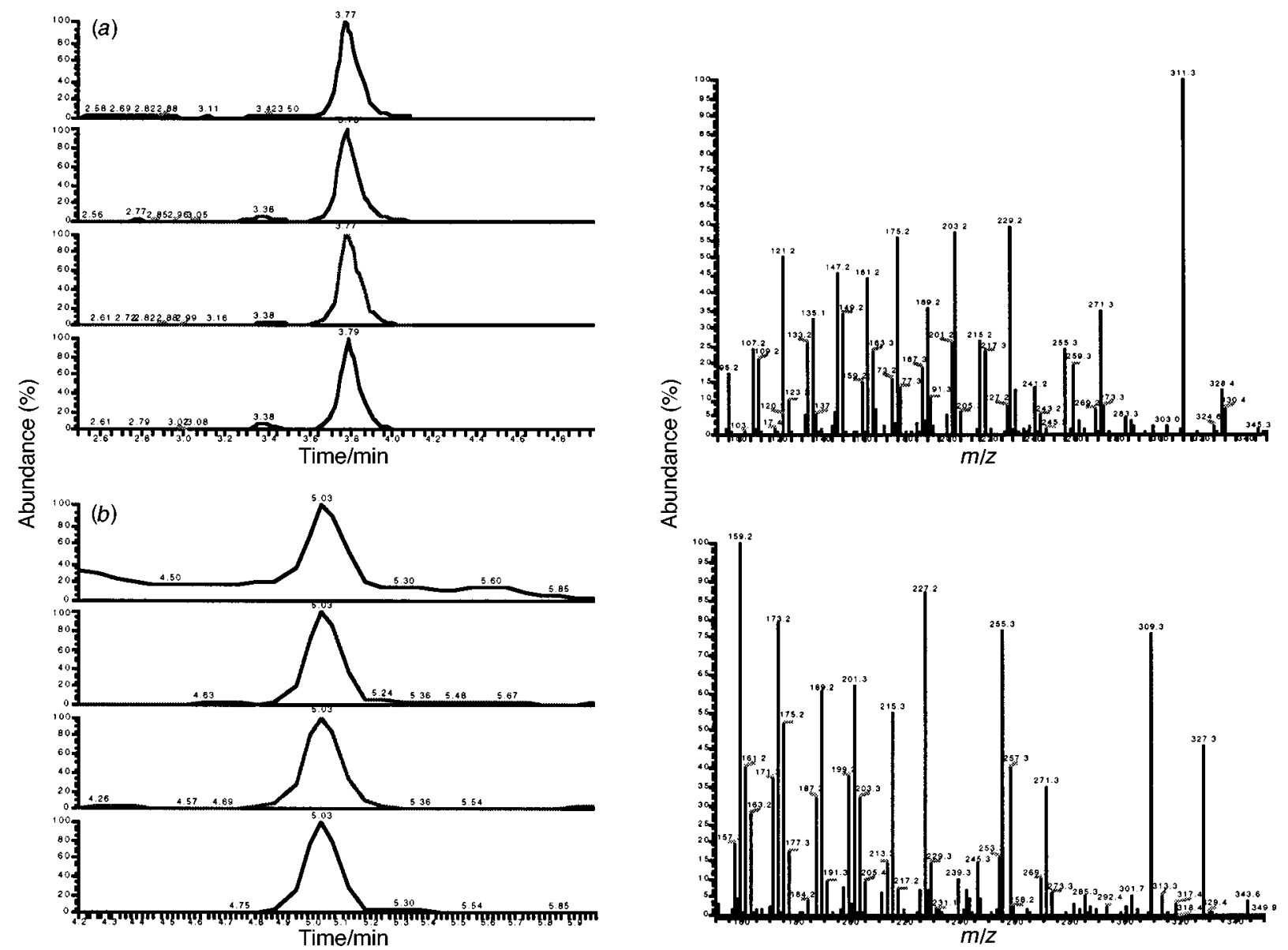

Fig. 6 Ion chromatogram and mass spectrum of 16-OHStan $(a)$ and Stan $(b)$ in the urine of animal 1321 taken $(a) 8 \mathrm{~d}$ and $(b) 3 \mathrm{~h}$ after injection (data ROL).

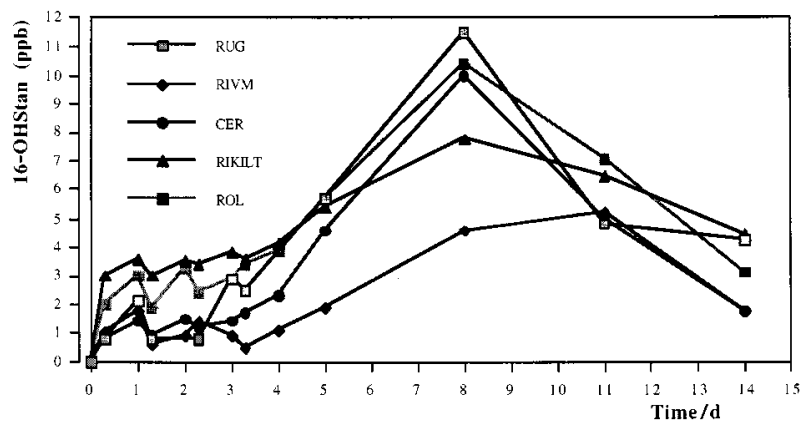

Fig. 7 Time course of 16-OHStan in urine following injection of $200 \mathrm{mg}$ of Stan into a veal calf.

possible to detect the metabolites, the laboratory reported that GC-MS of these components was difficult.

Hence, the question as to which analyte should be validated for veterinary inspection purposed was answered. The next step in this multi-laboratory study is a comparison of the advantages and disadvantages of all the methods with the aim of selecting the best method. The method selected may be validated subsequently.

\section{Acknowledgement}

At the meeting of the Stan working group the first author was chosen as the co-ordinator of the group. He thanks all his colleagues for their participation. The group also thanks Dr. R. Stephany, Director of the European Union Communities Reference Laboratory for Residues, for his interest in and support of this study.

\section{References}

1 EEC Council Directive 96/22, Off. J. Eur. Commun., 1996, L125, 3.

2 The Merck Index, 1996, 12th edn. on CD-ROM, version 12:1, Nr 8951, Chapman and Hall, London, 1996.

3 R. Verbeke, J. Chromatogr., 1979, 177, 69.

4 P. Teale L. Grainger and E. Houghton, presented at the 21st International Mass Spectrometry Conference, Amsterdam, August 26-30, 1991, poster TuM-CO2.

5 W. Schänzer G. Opfermann and M. Donike, J. Steroid Biochem., 1990, 36, 153.

6 W. Schänzer and M. Donike, Anal. Chim. Acta, 1994, 275, 23.

7 W. Schänzer, Ph. Delahaut, H. Geyer, M. Machnik and S. Horning, J. Chromatogr. B, 1996, 687, 93.

8 R. Massé, C, Ayotte, H. G. Bi and R. Dugal, J. Chromatogr. Biomed Appl., 1989, 497, 17.

9 H-Y.P. Choo, O.-S. Kwon, and J. S. Park, J. Anal. Toxicol., 1990, 14, 109.

10 M. W. Muck and J. D. Henion, Biomed. Environ. Mass Spectrom., 1990, 19, 37.

11 Ph. Delahaut, X. Taillieu, M. Dubois, K. De Wasch, H. F. De Brabander, P. Batjoens and D. Courtheyn, Arch. Lebensmittelh., 1998, 49, 3 .

12 V. Ferchaud, B. Le Bizec, M. P. Montrade, D. Maume, F. Monteau and F. André, J. Chromatogr B, 1997, 695, 269.

13 F. Smets, H. F. De Brabander, P. J. Bloom and G. Pottie, J. Planar Chromatogr., 1991, 4, 207.

14 P. Batjoens, H. F. De Brabander, F. Smets and G. Pottie, Analyst, 1994, 119, 2607.

15 K. Vanoosthuyze, E. Daeselaeire, A. Van Overbeke, C. Van Peteghem and A. Ermens, Analyst, 1994, 119, 2655.

16 L. Hendriks, B. Gielen, L. Leyssens and J. Raus, Vet. Rec., 1994, 134, 192.

17 Institute of Veterinary Inspection, Brussels, Belgium 1998, personal communication.

18 EEC Council Decision 93/256, Off. J. Eur. Commun., 1993, L118, 64.

Paper $8 / 05110 \mathrm{~K}$ 